Revue des patrimoines

\title{
L'utilisation du numérique, nouvelles perspectives pour la conservation-restauration des œuvres - Le cas particulier du site de la Carapa, Kourou, Guyane
}

Digital applications and new perspectives for the conservation and restoration of works of art; the case of the Carapa site at Kourou, French Guiana

\section{Anne-Laure Goron}

\section{(2) OpenEdition}

1 Journals

\section{Édition électronique}

URL : http://journals.openedition.org/insitu/22228

DOI : $10.4000 /$ insitu. 22228

ISSN : 1630-7305

Éditeur

Ministère de la Culture

\section{Référence électronique}

Anne-Laure Goron, «L'utilisation du numérique, nouvelles perspectives pour la conservationrestauration des œuvres - Le cas particulier du site de la Carapa, Kourou, Guyane », In Situ [En ligne], 39 | 2019, mis en ligne le 01 juin 2019, consulté le 11 juillet 2019. URL : http:// journals.openedition.org/insitu/22228 ; DOI : 10.4000/insitu.22228

Ce document a été généré automatiquement le 11 juillet 2019.

In Situ Revues des patrimoines est mis à disposition selon les termes de la licence Creative Commons Attribution - Pas d'Utilisation Commerciale - Pas de Modification 4.0 International. 


\section{L'utilisation du numérique,} nouvelles perspectives pour la conservation-restauration des œuvres - Le cas particulier du site
de la Carapa, Kourou, Guyane

Digital applications and new perspectives for the conservation and restoration of works of art; the case of the Carapa site at Kourou, French Guiana

\section{Introduction - la 3D à la portée de tous}

1 Dans un premier temps sceptique quant à l'utilité directe de la numérisation et modélisation 3D dans la pratique de notre métier, par définition ancré dans la matière, nous nous sommes néanmoins intéressée à cet outil et avons suivi une formation sur la modélisation $3 \mathrm{D}^{1}$ qui depuis nous a servi à plusieurs reprises, dans des optiques finalement assez diverses.

2 L'utilisation de logiciels de $\mathrm{PAO}^{2}$ tels qu'Illustrator, Photoshop ou encore InDesign est à présent largement répandue pour la réalisation de schémas, de relevés d'altération ou de mise en œuvre des objets qui nous sont confiés. On a même vu apparaître ces dernières années des logiciels de constats d'état sur support photo, tels que Horus Condition Report $^{\oplus 3}$ ou Art Report ${ }^{\oplus 4}$, pour répondre à la demande grandissante émanant des musées de disposer de constats d'états systématisés, plus fonctionnels, mais également réutilisables tout en étant sécurisés.

3 C'est donc maintenant la 3D qui fait son apparition dans notre domaine. Sans aller jusqu'à l'utilisation de l'imprimante 3D, qui reste sans doute un outil à explorer, la modélisation $3 \mathrm{D}$ elle-même, en dématérialisé avec le format de fichier pdf 3D, s'avère déjà un outil très 
utile. Nous avons pu l'exploiter dans le cadre de notre travail, pour la restitution d'éléments par modelage. Par exemple, il manquait les extrémités de la moustache d'un buste en plâtre de Jean Léon Gérôme par Jean-Baptiste Carpeaux ${ }^{5}$, conservé au ChâteauMusée de Nemours, dont il existe un autre exemplaire au musée du Petit Palais (Paris) ${ }^{6}$ (fig. 1).

Figure 1
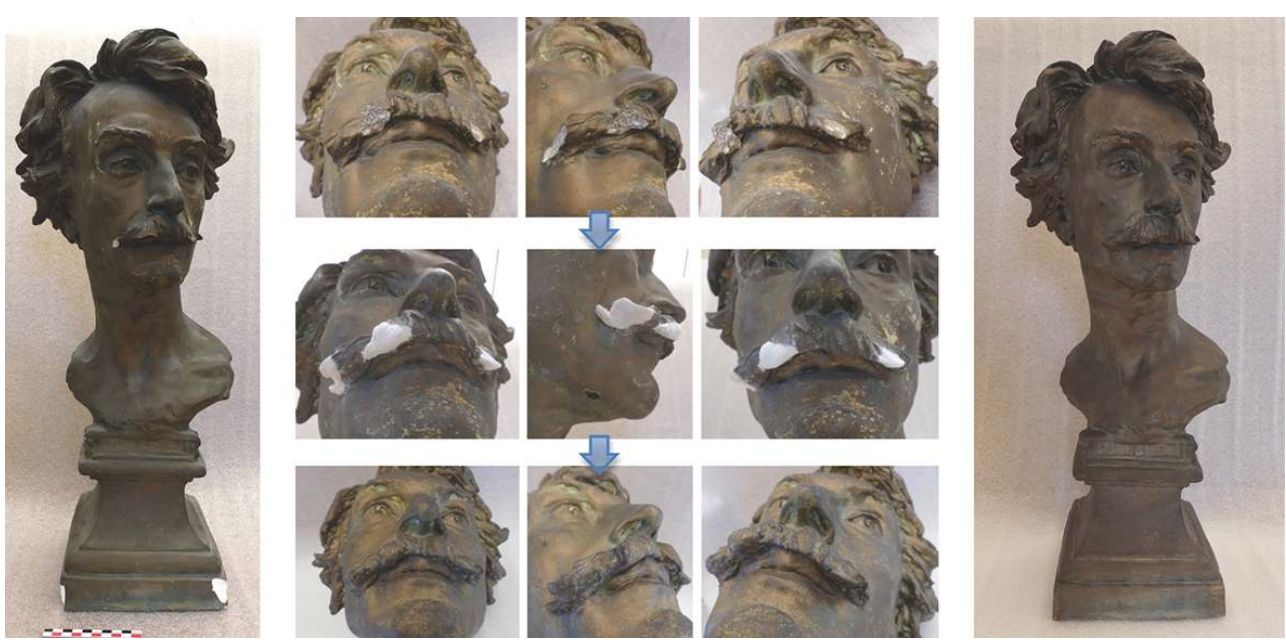

BUSTE DE J. L. GÉRôME PAR J.-B. CARPEAUX, AVANT INTERVENTION. DÉTAIL DE LA MOUSTACHE EN COURS D'INTERVENTION. LE MÊME BUSTE, APRÈS INTERVENTION.

PHOT. ANNE-LAURE GORON. ( ) ANNE-LAURE GORON.

4 Une simple demande auprès de la conservatrice de ce musée nous a permis d'obtenir quelques photos de son exemplaire, prises sur place selon certaines préconisations. Ces clichés ont alors pu être chargés dans un logiciel de photogrammétrie 3D et nous avons ainsi obtenu, dans ce cas précis moins de deux heures après la demande, la réplique de la moustache manquante en trois dimensions (fig. 2), sans même avoir eu à nous déplacer. $\mathrm{Au}$ lieu de réaliser le modelage de cet élément d'après photos (2D), nous pouvons nous appuyer sur un modèle 3D (fig. 3), qui, certes, n'est pas physiquement présent, mais que nous pouvons déplacer dans l'espace à volonté pour en comprendre la forme et la restituer le plus fidèlement possible. Dans ce cas précis, l'outil n'aura pas remplacé la main du restaurateur mais aura largement contribué à l'assister intellectuellement, lui évitant tout le travail de recomposition mentale de la forme. Le gain de temps est incontestable, et la fidélité de la forme mieux assurée. 
Figure 2

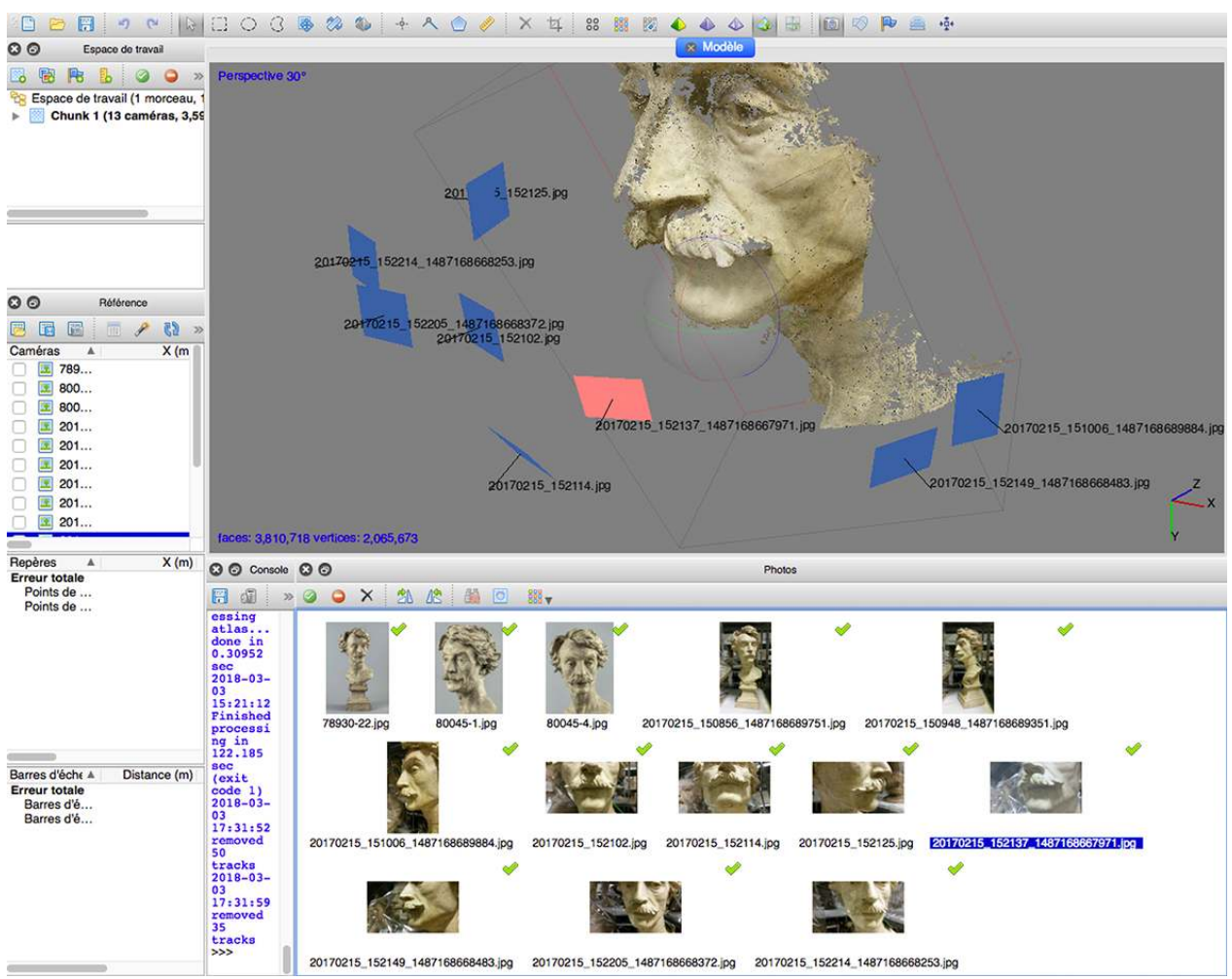

Modélisation d'une partie du Buste de J. L. Gérôme, d'après les photos fournies par le Petit Palais Logiciel Agisoft, version démo.

Phot. Anne-Laure Goron. ( ) Anne-Laure Goron. 
Figure 3

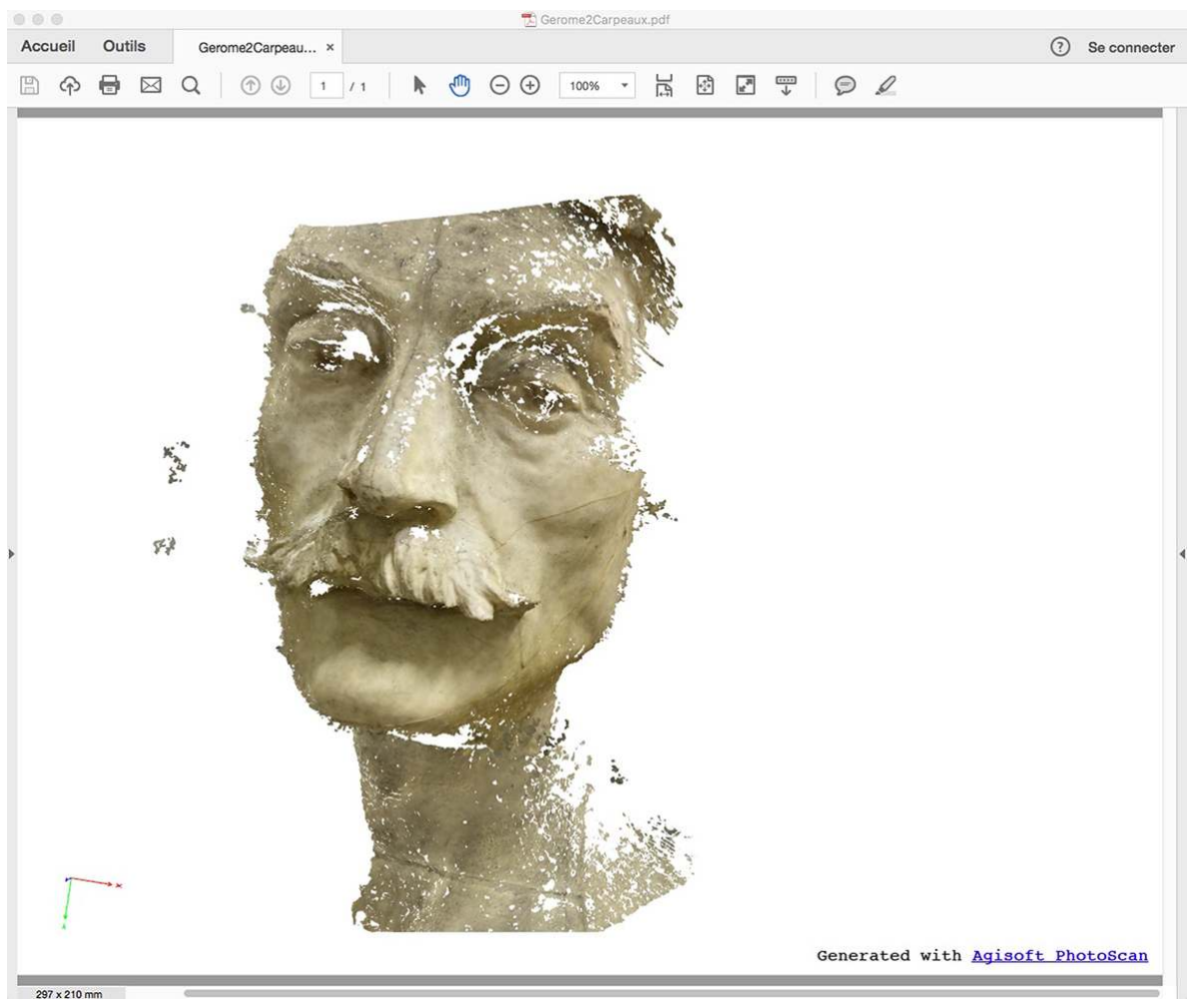

Sortie PDF-3D du modèle 3D d'une partie du Buste de J. L. Gérôme.

Phot. Anne-Laure Goron. (c) Anne-Laure Goron.

5 Cette expérience à petite échelle a constitué une bonne introduction à l'utilisation de la 3D pour d'autres problématiques de conservation-restauration, inhérentes au constat d'état de grande ampleur.

\section{Les roches gravées de la Carapa}

\section{Présentation d'un site exceptionnel}



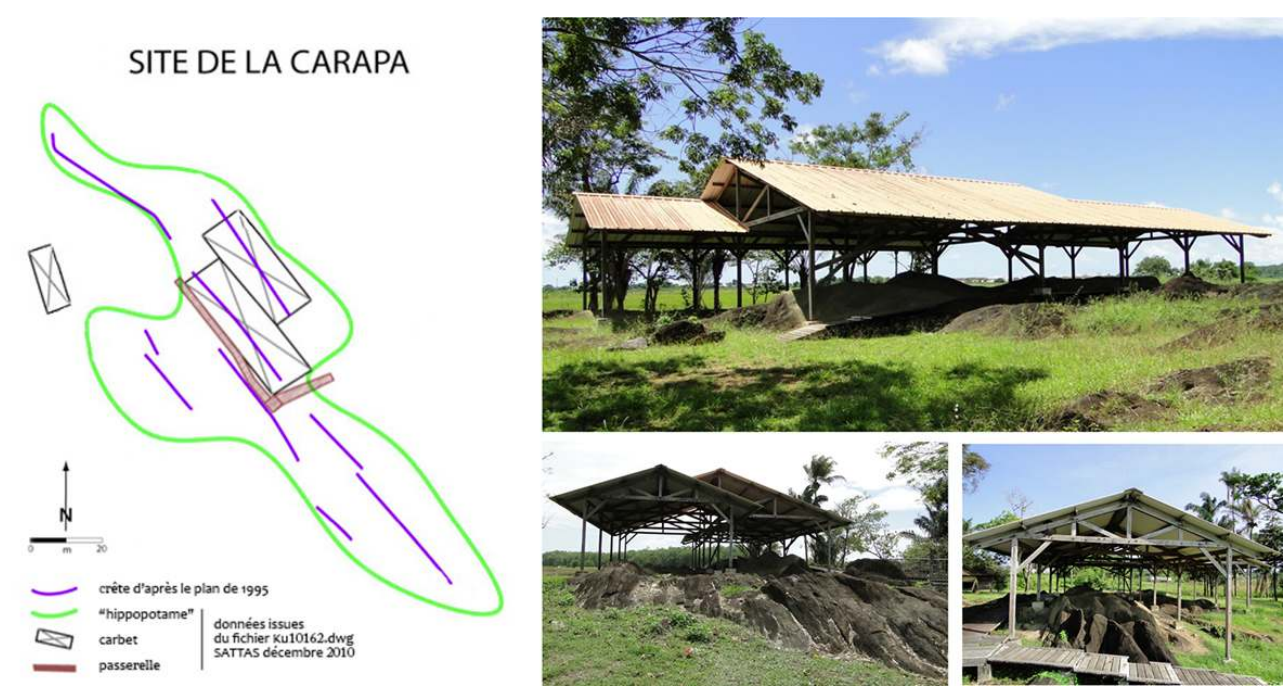

Site de la Carapa, vue des roches gravées en arrivant du CAAK nord-est, vue nord-ouest et vue sud-est de la partie des roches recouvertes par le carbet.

Schéma P. Boivin. (c) Pierre Boivin. Phot. Anne-Laure Goron. (c) Anne-Laure Goron.

6 Le site de la Carapa (fig. 4) est un affleurement rocheux, autrement appelé «savaneroche » ou encore « hippopotame de savane», s'étendant sur une centaine de mètres de long et une cinquantaine de mètres de large, et pouvant s'élever jusqu'à $2 \mathrm{~m}$ de haut par endroit. Ce n'est qu'en 1992, grâce à un extrait de plan cadastral daté de 1904 où figurait la mention "Roches gravées", que ce site a pu être redécouvert, et enfin étudié (puis classé monument historique en 1993) ${ }^{7}$. Il était alors recouvert d'une végétation abondante, qui a dû être dégagée pour les besoins d'observation des scientifiques. Soucieux du traumatisme que pourrait entraîner la mise à nue de la roche et notamment les effets du soleil direct, les services de la DAC (Direction des Affaires culturelles) ont décidé de la protéger au moins partiellement, par la mise en place d'un « carbet » (abri de bois sans murs typique des cultures amérindiennes) ${ }^{8}$ (fig. 5).

Figure 5

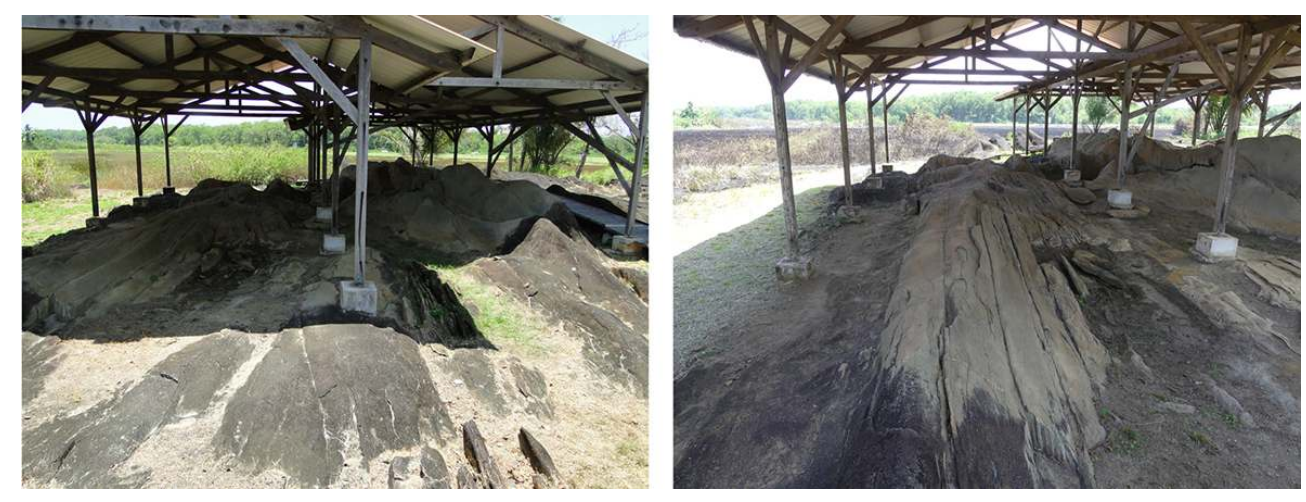

Site de la Carapa, vues nord-ouest et sud-est des roches gravées situées sous le carbet.

Phot. Anne-Laure Goron. (c) Anne-Laure Goron.

7 La roche elle-même est une ancienne lave basaltique transformée en amphibolite à talc et chlorite. Ces deux derniers minéraux très tendres expliquent que la roche soit douce au 
toucher et facile à graver'. Son hétérogénéité est également à l'origine de vasques naturelles retenant les eaux météoriques (fig. 6), dont l'homme a tiré profit en aménageant à proximité des zones de polissage (polissoirs de forme ovale ou à rainure) ${ }^{10}$.

Figure 6

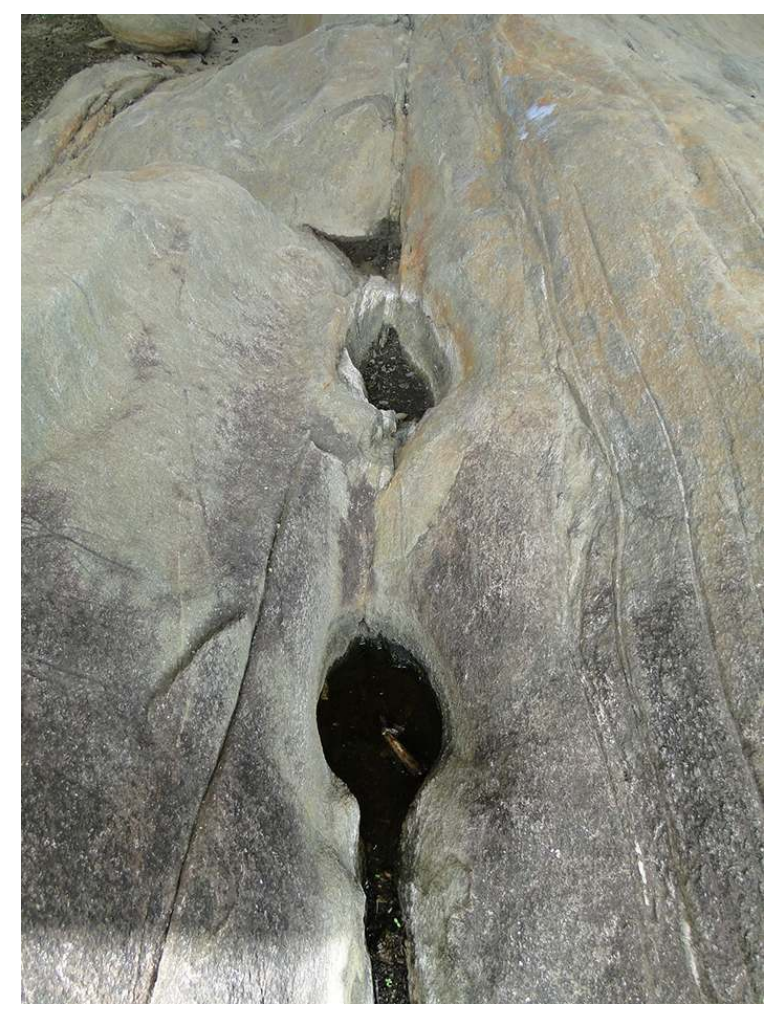

Site de la Carapa, exemple d'une série de vasques naturelles.

Phot. Anne-Laure Goron. (c) Anne-Laure Goron.

8 Mais ce sont les gravures en surface de cette roche (fig. 7) qui font de ce site un témoignage exceptionnel des populations amérindiennes précolombiennes de Guyane : au total, 248 figures ${ }^{11}$ ainsi que de nombreuses cupules ont été incisées par abrasion ou par percussion dans l'épiderme de la roche (fig. 8). Plus de $70 \%$ d'entre elles sont des figures anthropomorphes, les autres étant des formes zoomorphes, ou géométriques qui n'ont pas toujours pu être identifiées. Elles ont néanmoins en commun une construction systématique, à base de modules simples construits à partir de lignes, conférant à ce site une grande unité stylistique, qui témoigne d'une homogénéité culturelle. 
Figure 7
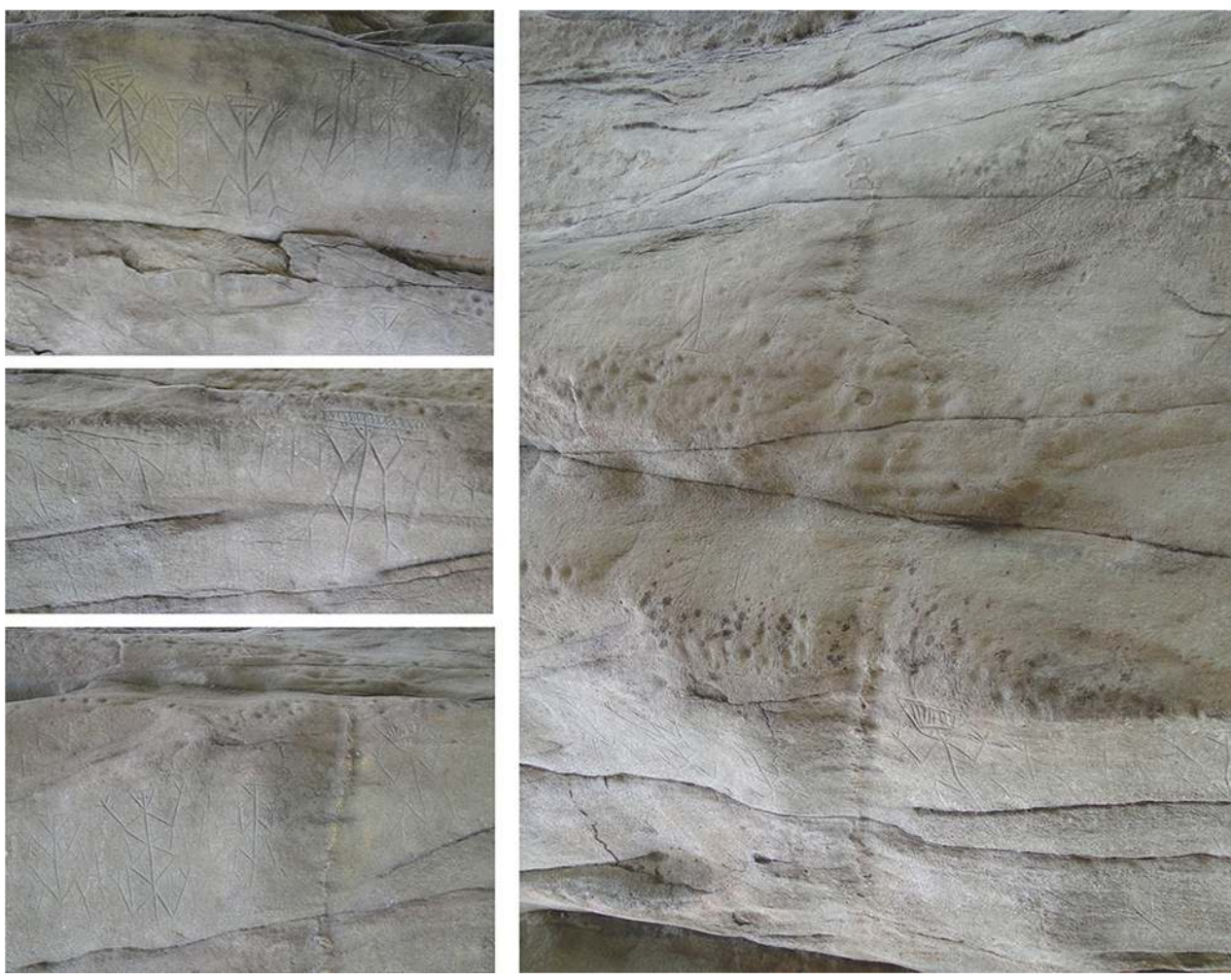

Site de la Carapa, exemples de gravures et d'une zone de cupules. On constate que les gravures sont plutôt situées sur les parties hautes des flancs de la roche, et les cupules plutôt localisées le long des crêtes.

Phot. Anne-Laure Goron. () Anne-Laure Goron.

\section{Figure 8}
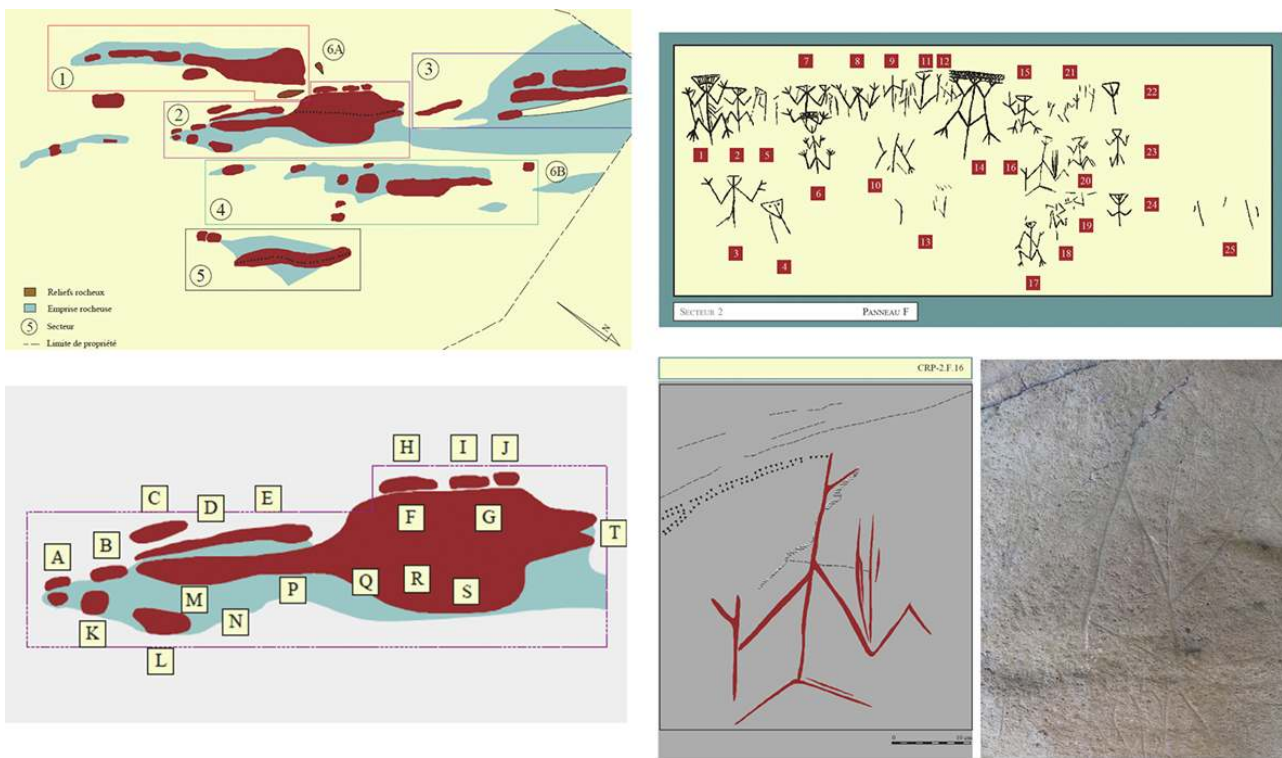

Extraits du rapport de N. Aujoulat : plan du site figurant les différents « secteurs » de gravures, et détail du Secteur 2 ; détail du « panneau » de gravures du secteur 2 ; détail d'une figure du panneau $\mathrm{F}$,

également visible sur l'un de nos relevé (R23) - c'est cette zone qui a été choisie pour tester le logiciel Aïoli.

Schémas N. Aujoulat. @ CNP. Phot. Anne-Laure Goron. @ Anne-Laure Goron. 
En l'absence de matériel archéologique, les historiens ont à ce jour trop peu d'éléments pour dater précisément les gravures ou en déterminer le contexte exact. L'hypothèse la plus partagée serait qu'il s'agit d'un site cérémoniel, pouvant dater du $1^{\mathrm{er}}$ millénaire de notre ère, occupé relativement longtemps ou du moins de façon répétée.

\section{Problématique / Demande}

10 Suite à la redécouverte et à la dé-végétalisation du site, des inquiétudes ont surgi sur l'évolution de l'état des roches, les choix effectués jusqu'alors et leur impact sur la bonne conservation du site, qui se retrouve à présent entouré d'une zone industrielle, implantée relativement récemment malgré les restrictions dues à son classement.

11 Ce fut le point de départ d'une réflexion collégiale des services patrimoniaux de la DAC ${ }^{12}$ de Guyane et de divers spécialistes, autour du sujet des roches gravées de Guyane en général, et plus particulièrement de ce site majeur.

12 Celui-ci est à présent ouvert au public, par le biais de visites guidées et contrôlées, organisées par le $\mathrm{CAAK}^{13}$ (Centre d'archéologie amérindienne de Kourou), construit à quelques centaines de mètres du site et inauguré fin 2013. Le site fait par ailleurs l'objet de plusieurs projets d'aménagement, qui, le touchant directement ou indirectement, auront un impact non négligeable sur les roches. La nécessité de réaliser un état zéro qui pourrait constituer une référence pour la conservation du site est devenue impérative. Ce travail a été confié à une équipe inter-disciplinaire, constituée de géologues du CNRS et d'un conservateur-restaurateur ${ }^{14}$, grâce au financement intégral de la DAC de Guyane. Dans ce cadre, et avec la collaboration du CNP ${ }^{15}$ (Centre national de préhistoire), nous avons pu tester la plateforme «Aïoli $»^{16}$ élaborée par le Laboratoire MAP ${ }^{17}$ (Modèles et simulations pour l'architecture et le patrimoine) UMR CNRS/MC $\mathrm{n}^{\circ} 3495$, et évaluer son intérêt pour nos travaux de conservation-restauration sur le site de la Carapa.

\section{Le suivi sur support 2D : Photoshop ${ }^{\circledR}$, ses avantages, ses limites}

\section{Objectif : un outil de suivi utilisable sur site et par tous}

13 En tant que conservateur-restaurateur de l'équipe, notre principal rôle était d'établir une cartographie des altérations visibles des roches, qui formerait le point de départ d'un suivi exploitable par tous les scientifiques en lien avec le sujet, qu'ils soient conservateurs, archéologues, géologues ou restaurateurs. Il fallait également que cet outil soit «mobile ", c'est-à-dire transportable sur site, le principe du suivi étant de comparer le constat d'origine, dit constat à $t_{0}$, avec la situation actuelle. Il fallait donc pouvoir revenir avec l'outil devant un point précis du site, pour statuer sur son évolution.

L'élaboration d'un constat d'état fiable et global s'est révélée compliquée à mettre en place, du fait de la morphologie de la roche - site vaste, nombreux plis de la roche -, de la localisation éparse des gravures et de la présence d'un carbet empêchant une vue d'ensemble prise du dessus. Par ailleurs, les logiciels disponibles actuellement ne sont pas encore adaptés à des «objets " de cette dimension, ni à un constat d'état aussi détaillé. Nous avons donc travaillé à partir de la méthode désormais «classique » consistant à annoter une photo, autrefois à la main, aujourd'hui sur support numérique. 


\section{Méthode : relevé sous Photoshop ${ }^{\circledR}$ et suivi par photos macros}

\section{Élaboration d'un support}

15 La première étape consistait à mettre en place le support numérique lui-même. La taille et la complexité du site obligent à multiplier les prises de vues qui sont la base de nos relevés. Nous n'avions même pas de vue aérienne sur laquelle nous appuyer puisque le site est couvert par le carbet.

16 Ces problèmes techniques nous ont conduite à nous intéresser aux outils numériques 3D. Des relevés photogrammétriques avaient déjà été effectués sur ce site et exploités par l'un des membres de l'équipe, qui nous a fourni une ortho-photographie (fig. 9). Celle-ci étant encore trop lacunaire, et de toute façon, trop lourde pour nos outils informatiques, nous n'avons pas pu l'utiliser comme support pour les relevés eux-mêmes. Elle nous a cependant été utile au cours de notre travail, notamment pour nous repérer sur la roche, lorsque nous étions en dehors du site.

Figure 9

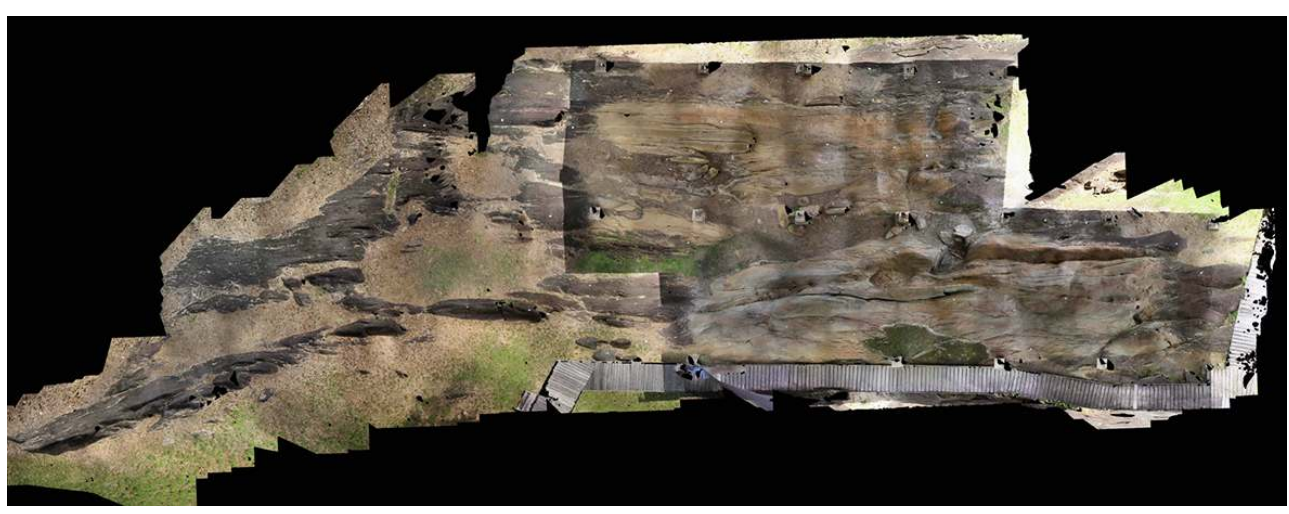

Ortho-photographie des roches gravées de la Carapa.

Phot. Franck Vautier. (c) Franck Vautier.

17 Pour l'élaboration du support général de relevé, nous avons donc effectué une série de photos, représentant chacune une parcelle d'environ $2 \mathrm{~m}^{2}$, afin de couvrir le site de la façon la plus exhaustive possible, c'est-à-dire en prenant en compte les nombreux plis de la roche et les zones encaissées ${ }^{18}$. Le résultat est une sorte de mosaïque photographique de tout le développé du site (fig. 10), restreinte toutefois à la zone couverte par le carbet pour des raisons de temps. Une comparaison avec les zones hors carbet, de même que les faciès d'altération situés à la limite intérieur/extérieur du carbet, nous intéressant également, quelques-unes de ces zones ont fait l'objet d'observations comparatives ponctuelles. 
Figure 10

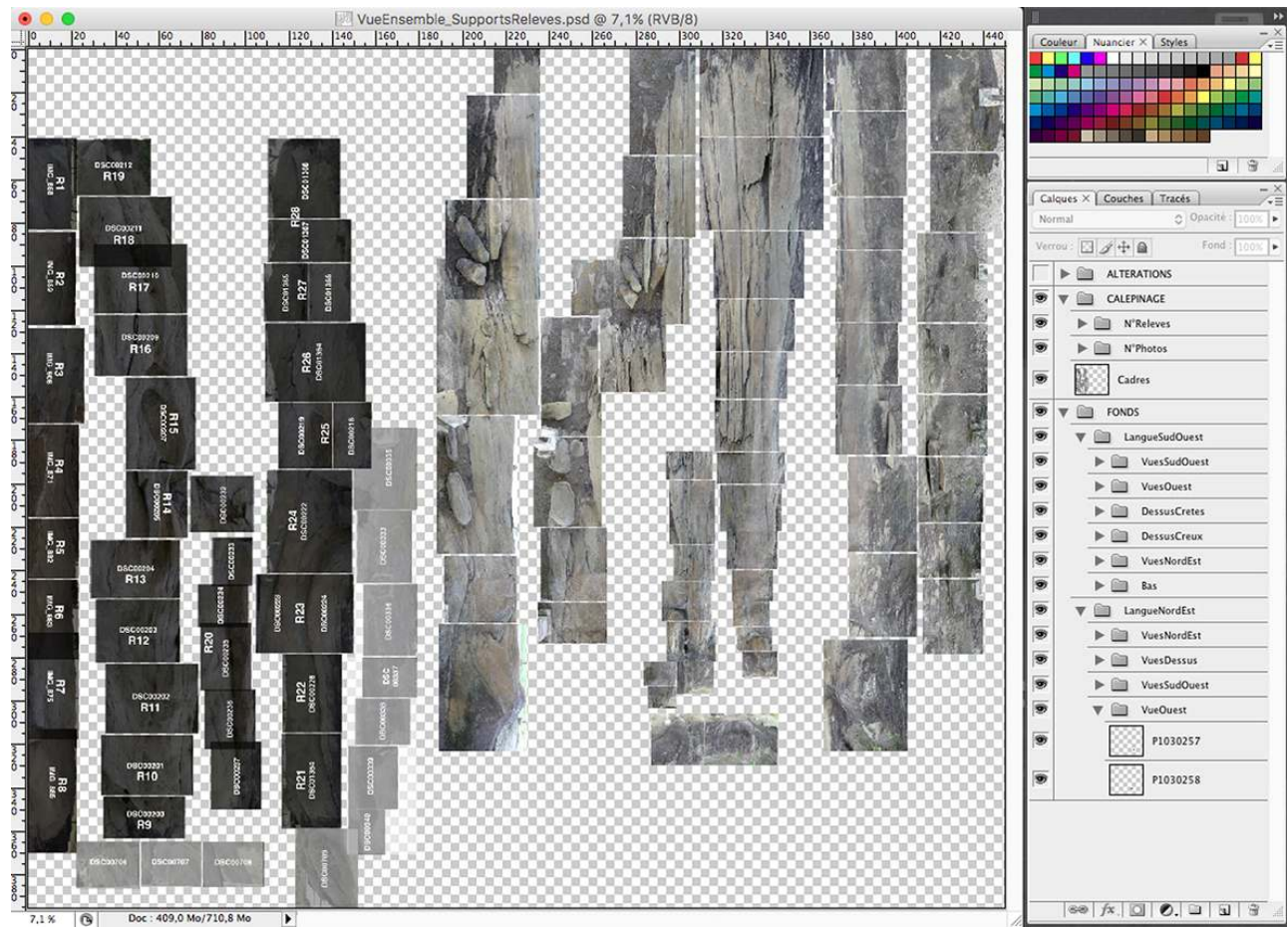

Le support général de relevé constitué d'une mosaïque de photographies, avec indication (pour la moitié du site) du numéro de relevé et numéro de la photographie ayant servi de support à ce relevé. Phot. Anne-Laure Goron. ( ) Anne-Laure Goron.

\section{Relevé sur tablette numérique}

L'établissement du constat d'état en tant que tel a alors pu commencer. Une phase d'observation a d'abord été nécessaire pour caractériser les faciès d'altération en présence et définir un glossaire ${ }^{19}$. Une liste de termes a été établie pour chaque faciès, accompagnés d'une description détaillée et d'une illustration. Les altérations observées in situ ont ensuite été minutieusement relevées sur chaque photo-support par le biais du logiciel Photoshop ${ }^{\circledast}$ Elements, sur une tablette numérique (fig. 11). Ce logiciel était le seul à notre connaissance qui soit adapté à nos besoins, puisqu'il permettait l'utilisation de calques et d'un grand choix d'outils : variation de l'épaisseur et de la couleur du trait; insertion de texte ; insertion de formes, notamment de cadres, pour la localisation rapide des photos macros, comme nous le verrons plus loin. 
Figure 11

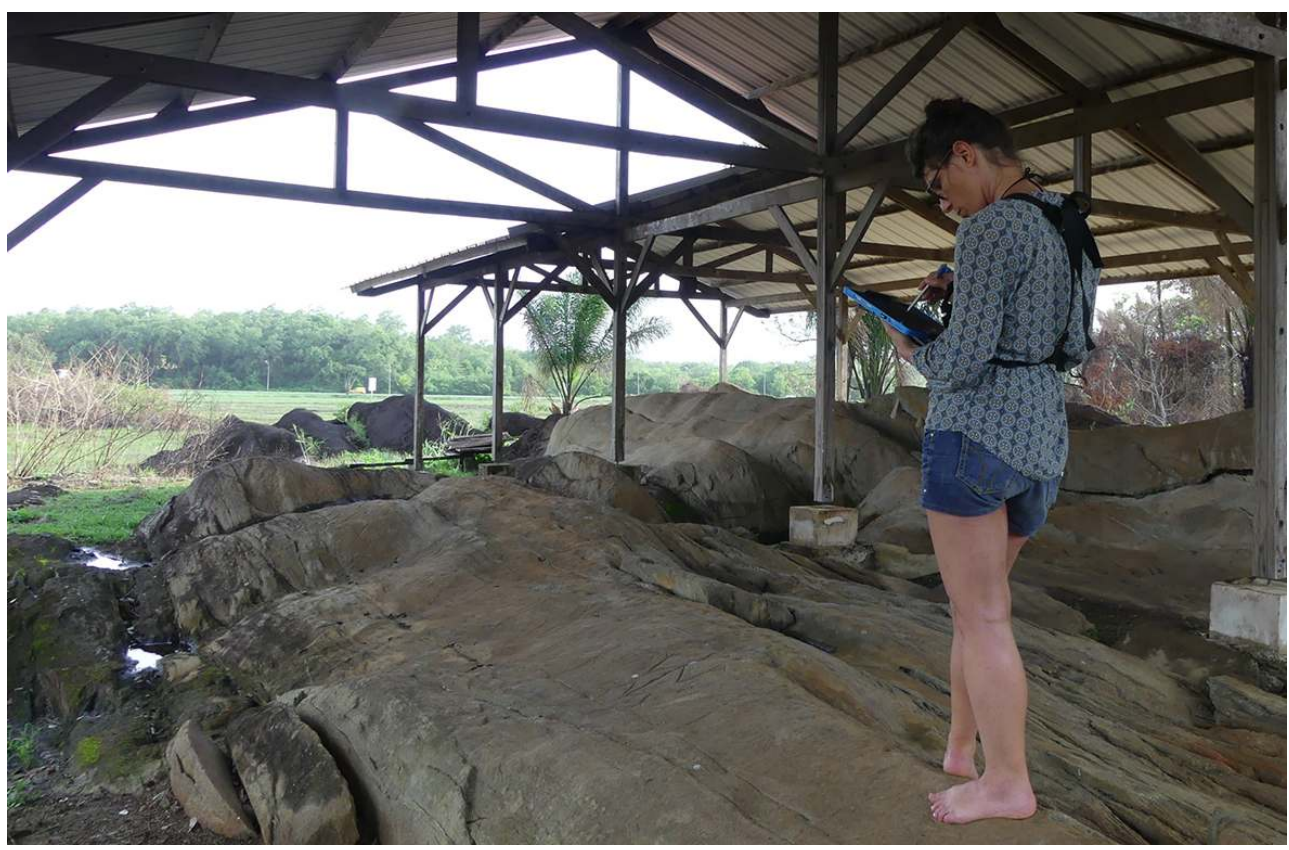

Le relevé sur site sur tablette numérique. On notera au passage que celle-ci est fixée sur un harnais afin de limiter les risques musculo-squelettiques.

Phot. Bérénice Valot. @ Bérénice Valot.

19 Disposer de calques était essentiel pour la bonne marche de notre travail car ils permettaient d'annoter les altérations séparément les unes des autres (fig. 12), tout en laissant la possibilité de les visualiser toutes en même temps pour faire apparaître les liens qu'il pouvait y avoir entre elles, ou au contraire individuellement afin d'améliorer la lisibilité lorsqu'on s'attachait à un type d'altération en particulier (fig. 13). 
Figure 12

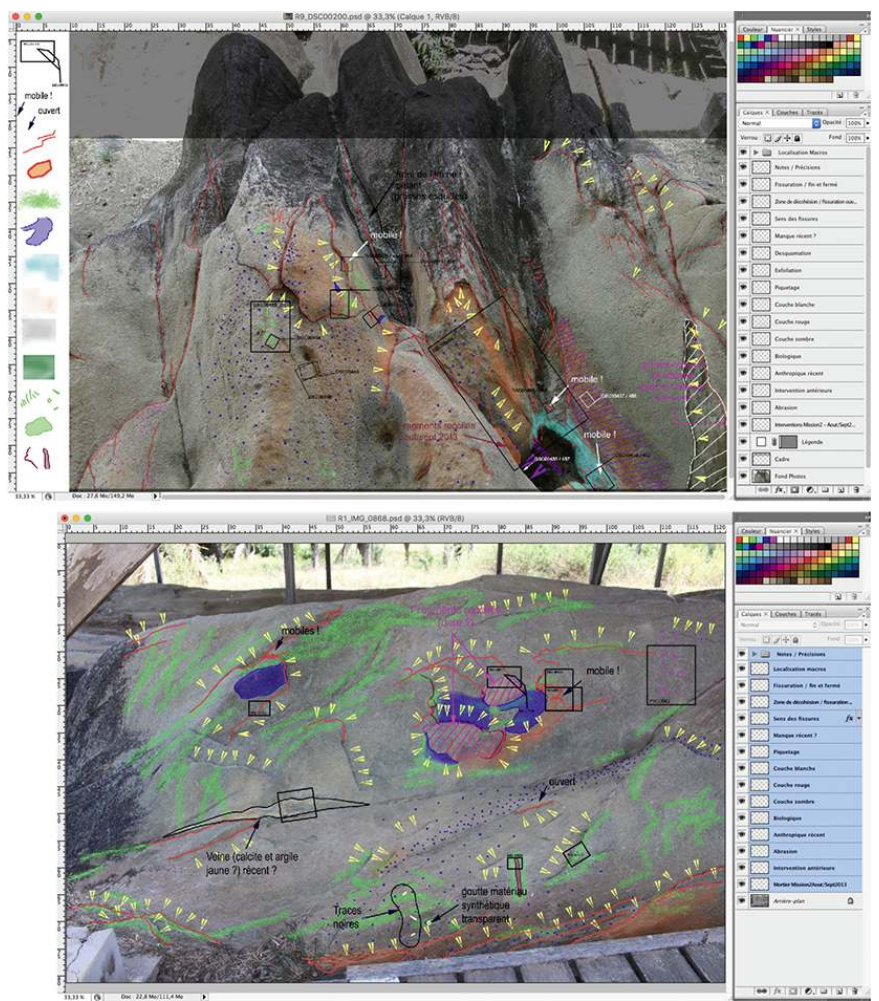

Exemples de deux relevés ( $R 1$ et $R 9$ ) réalisés sur Photoshop ${ }^{\circledR}$ Elements, tous calques visibles. Phot. Anne-Laure Goron. @ Anne-Laure Goron.

Figure 13

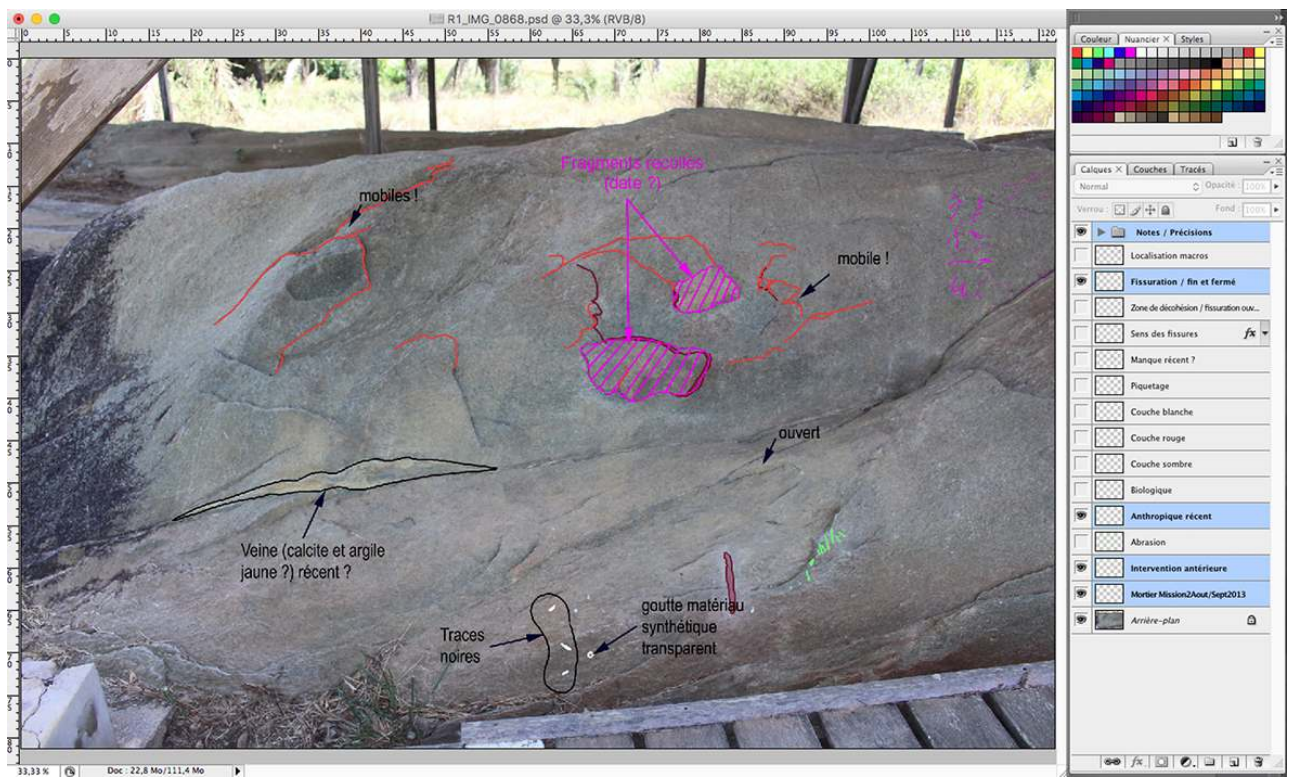

Exemple du relevé R1 réalisé sur Photoshop® Elements, avec 5 calques visibles sur 16. Phot. Anne-Laure Goron. (c) Anne-Laure Goron.

Pour chaque relevé, un échantillon des altérations a été illustré plus précisément par des macro-photographies - entre 5 et 15 par relevé - également localisées de façon précise 
sur les supports (fig. 14), et qui ont quant à elles un véritable rôle de témoin visuel à $t_{0}$ pour comparaison et suivi au cours du temps. Pour faciliter le repérage de ces zones, une « suite zoomée » - du plan large à un plan plus resserré pour arriver enfin à la macro - a parfois été réalisée.

Figure 14
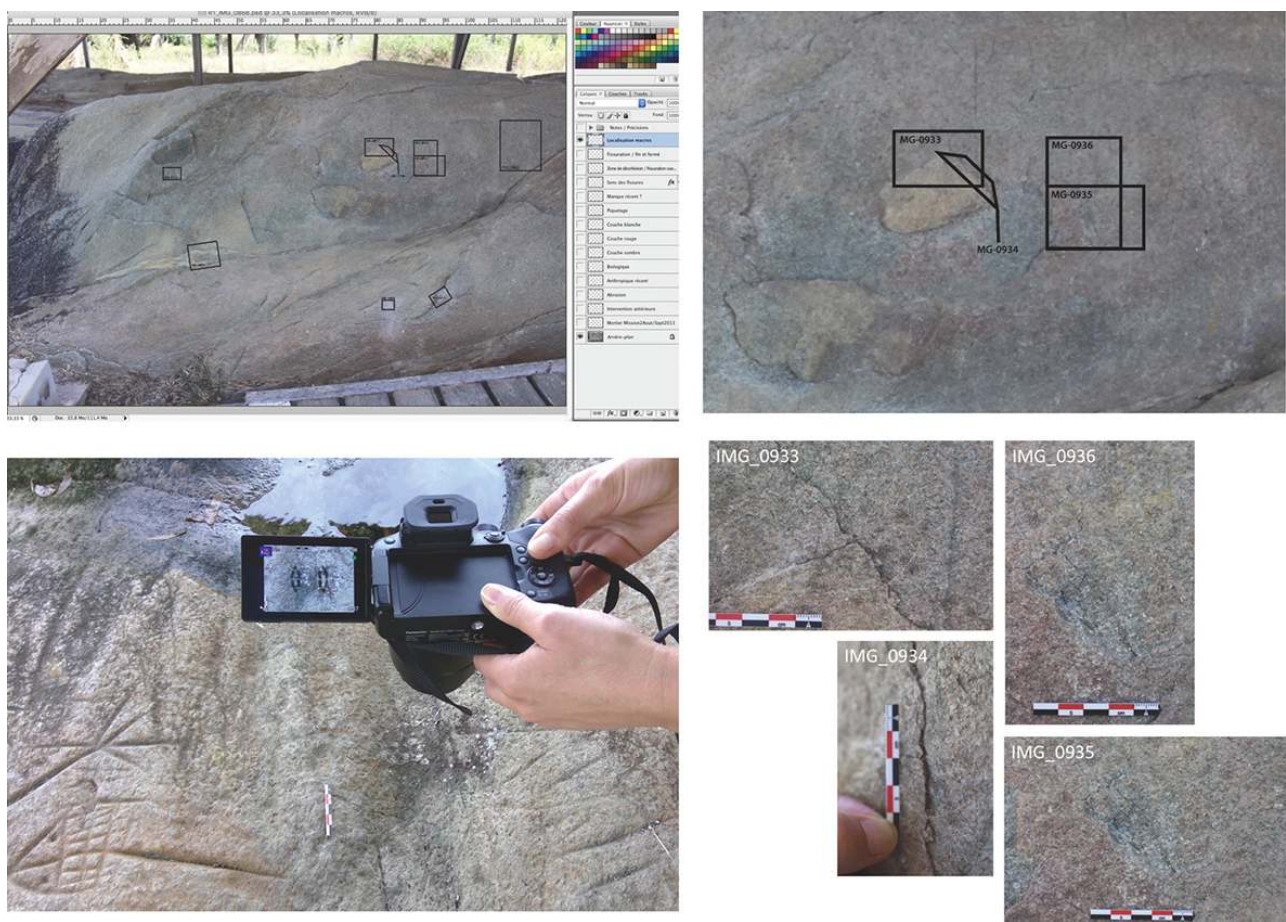

Le suivi par photo macros. Exemple du relevé $\mathrm{R} 1$ réalisé sur Photoshop® Elements, calque «localisation photos » visible et détail d'une partie de ce relevé figurant le cadrage de 4 photos macros.

Phot. Anne-Laure Goron. (c) Anne-Laure Goron.

Les photos elles-mêmes ont été indexées sur un tableur Excel ${ }^{\circledR}$, et classées dans des dossiers portant le nom du relevé. Elles pourront être retrouvées simplement par leur numéro, ce dernier étant également reporté sur le relevé lui-même à côté du cadre de localisation, dans l'optique de faciliter le suivi ultérieur (comparaison entre la photo témoin à $\mathrm{t}_{0}$ et l'existant au moment $\mathrm{t}_{+1 \text { an }}$ par exemple). Enfin, des analyses climatiques, de sels et de microbiologie, actuellement en cours, viendront compléter cette étude et affiner les hypothèses.

\section{Résultats}

Pour couvrir la totalité du site, une centaine de photos de fonds regroupées en une trentaine de relevés et près de 600 photos macros supplémentaires localisées sur ces mêmes relevés ont été nécessaires. Cette méthode nous a permis d'obtenir des relevés suffisamment précis pour notre objectif. Ceux-ci sont modifiables et pourront donc être amendés dans le cadre du suivi : toute nouvelle dégradation pourra être indiquée sur un nouveau calque et mise en relation avec les précédentes observations.

Mais pour avoir une vue globale du site, encore faut-il « lier » tous ces relevés entre eux, c'est-à-dire reporter les calques de tous les relevés sur la mosaïque de photographies. Une 
fois tous les calques des relevés visibles sur un seul document, nous espérons mieux comprendre les processus de dégradation et les liens potentiels entre eux. Cette synthèse des informations a montré ses limites dans le cas de la Carapa: l'étape de fusion des documents est fastidieuse et chronophage; comment gérer le poids du fichier .psd une fois assemblée la trentaine de relevés comprenant chacun une dizaine de calques; comment, enfin, traiter les parties qui se chevauchent pour qu'elles ne parasitent pas la lecture de l'ensemble?

\section{Le suivi sur support 3D : Aïoli, ses limites, ses perspectives}

\section{Les perspectives du relevé 3D} photogrammétrie, le principe d'Aïoli est de spatialiser et propager les annotations sur les images en se basant sur des correspondances 2D/3D. Ainsi, lorsque l'on annote une des images sources, le logiciel reporte automatiquement ces annotations sur les autres images, dont il connaît les points homologues, tout en générant la région spatiale relative à l'annotation, visualisable et manipulable en 3D. 


\section{Figure 15}

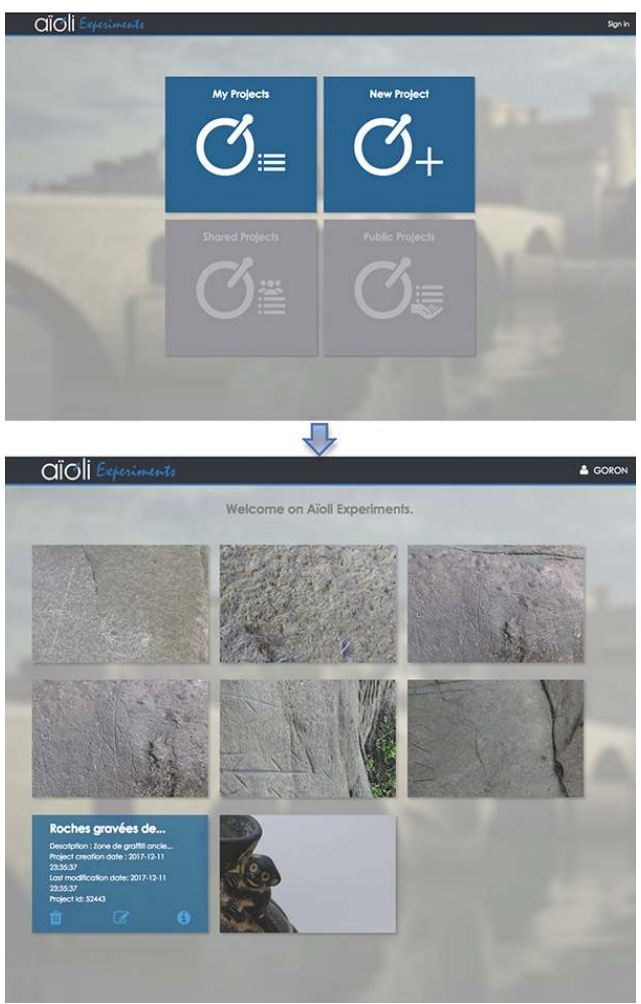

Plateforme Aïoli, capture d'écran de la page d'accueil après identification des projets en cours Campagne de béta-testing (période octobre 2017 - février 2018).

Phot. Anne-Laure Goron. (C) CNRS-MAP.

Pour accéder à Aïoli, il suffit de se connecter à la plateforme en ligne (service d'informatique en nuage - cloud computing) avec ses identifiants (fig. 15). Il s'agit ensuite de créer un "projet» et de charger les photos, qui auront été prises en amont en respectant le processus photogrammétrique ${ }^{22}$ adapté. Le logiciel traite ces images en les envoyant sur les serveurs de calcul distants et crée un modèle 3D. Lorsque le projet est " édité », toutes les images téléchargées apparaissent dans une fenêtre et la vue 3D dans une autre, sous la forme d'un nuage de points (fig. 16). 
Figure 16

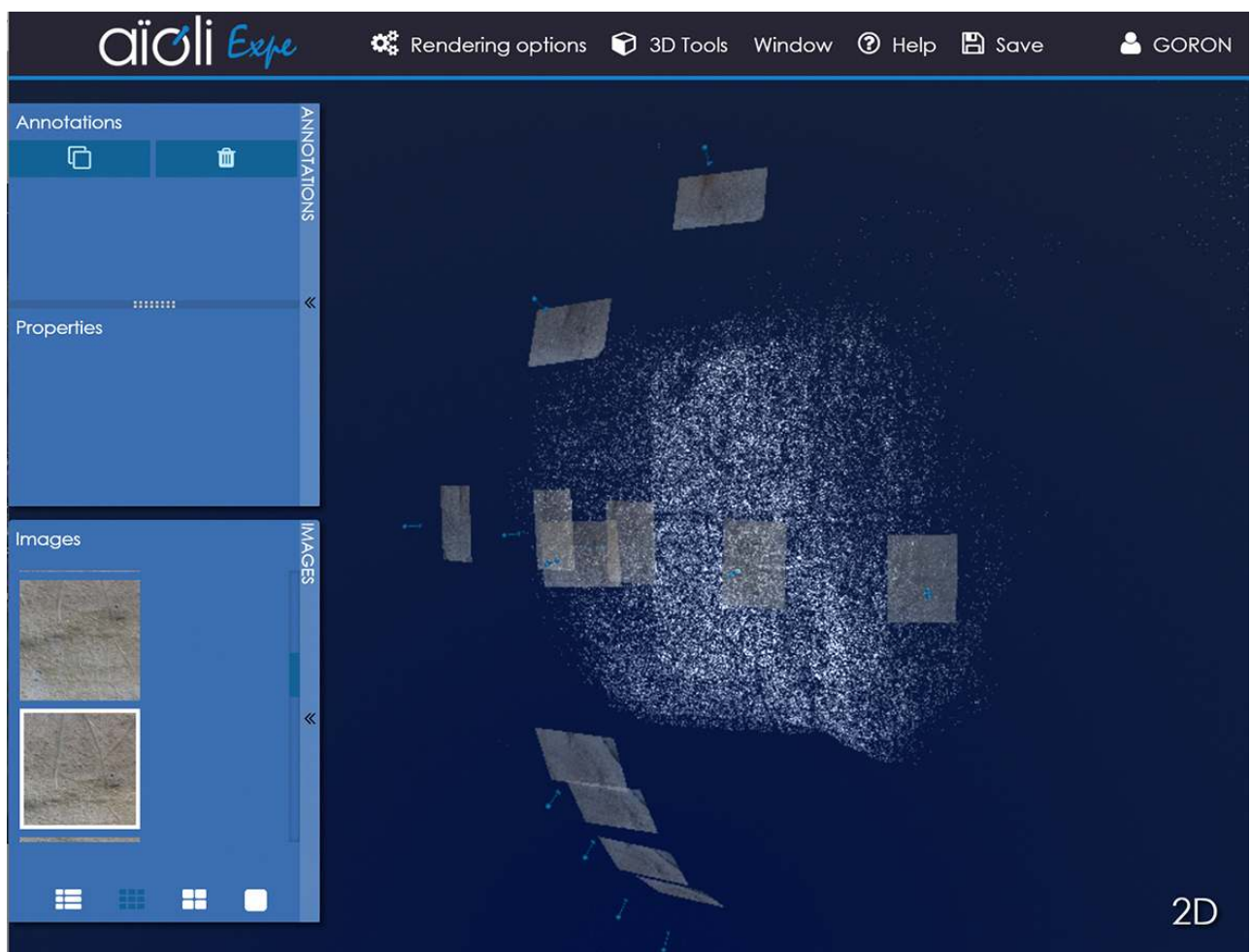

Plateforme Aïoli, capture d'écran d'un projet en cours. Campagne de béta-testing (période octobre 2017 - février 2018).

Phot. Anne-Laure Goron. (C) CNRS-MAP.

29 Il suffit alors de cliquer sur l'une des images, de créer un calque d'annotation pour y inscrire les observations grâce aux outils proposés (fig. 17), puis de valider. Le logiciel se charge de traiter ces nouvelles données et de les inclure dans le projet (fig. 18). Ces opérations peuvent être répétées autant de fois que nécessaire. 
Figure 17

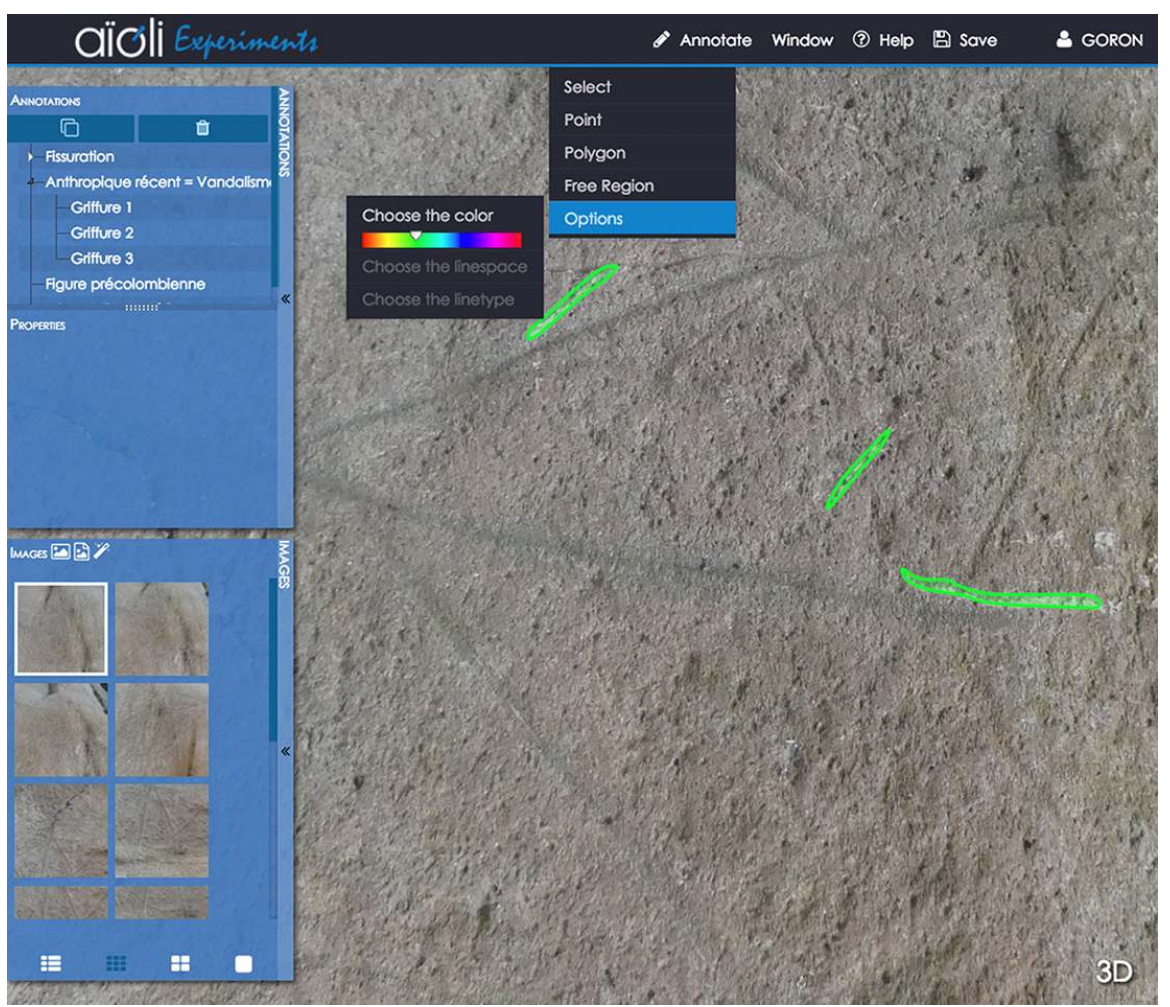

Plateforme Aïoli, capture d'écran d'un projet dont l'annotation est en cours. Campagne de béta-testing (période octobre 2017 - février 2018).

Phot. Anne-Laure Goron. (C) CNRS-MAP. 
Figure 18
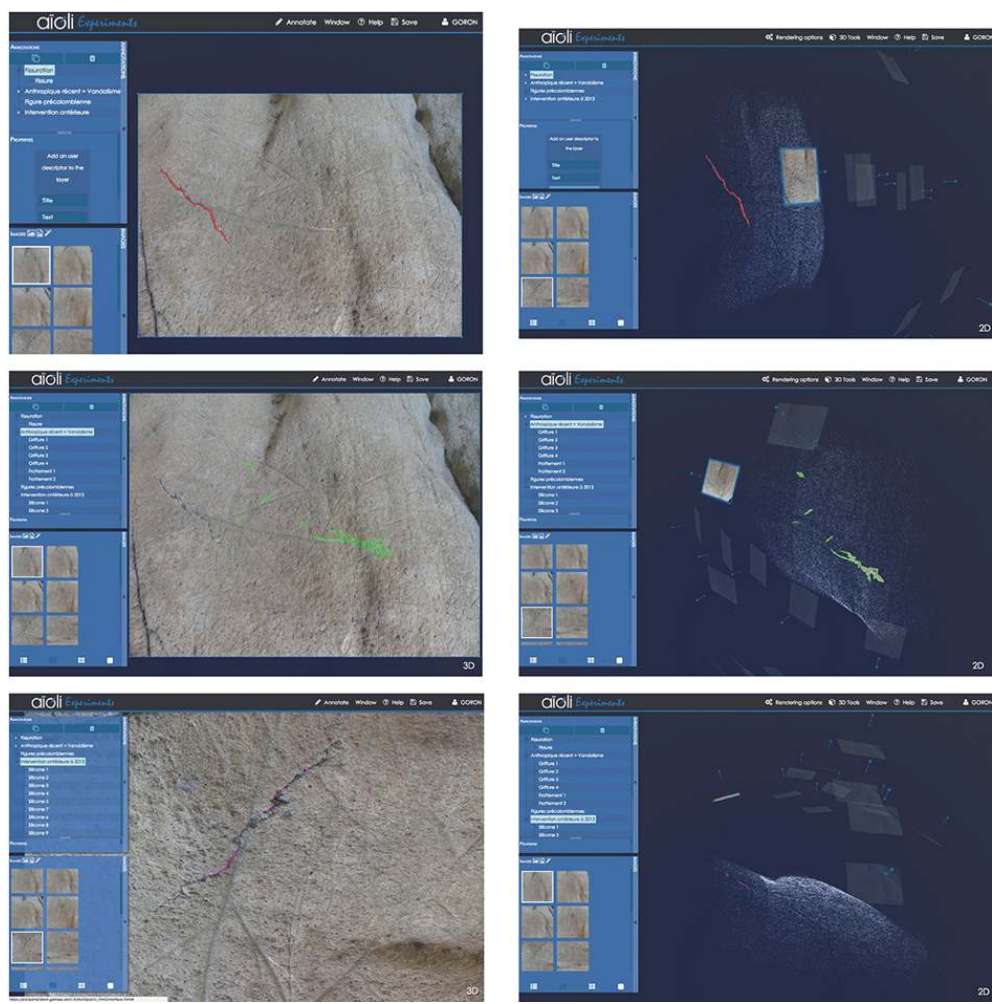

Plateforme Aïoli, captures d'écrans du même projet en cours. Exemple de 3 calques, avec à gauche la vue en 2D (relevé effectué sur la photo) et à droite le même relevé à chaque fois incrémenté dans le modèle en 3 dimensions. Campagne de béta-testing (période octobre 2017 - février 2018).

Phot. Anne-Laure Goron. ( $)$ CNRS-MAP.

Le modèle 3D peut ensuite être extrait sous la forme d'un fichier d'archive .zip, puis ouvert dans un logiciel libre tel que MeshLab, par exemple.

\section{Retour d'expérience}

Notre utilisation du logiciel s'est faite dans le cadre de la campagne de bêta-test, qui s'est déroulée d'octobre 2017 à février 2018. Nous avons donc pu le tester sur site lors de notre dernière mission guyanaise, en novembre-décembre 2017.

Le logiciel étant dépendant d'internet, et notre connexion «nomade » trop limitée, il ne nous a pas été possible de l'utiliser devant la roche elle-même. Cela ne nous pas empêchée d'exploiter ses outils au maximum et de suggérer des améliorations possibles.

En l'état actuel de l'outil, toutes nos attentes n'étaient pas comblées, mais nous avons déjà pu entrevoir ses capacités, que nous jugeons prometteuses. La possibilité d'éditer des calques répond à l'un de nos besoins fondamentaux, à savoir distinguer les altérations les unes des autres. Mais ce système de calques nous parait encore trop cloisonné : chaque annotation génère une région - associée à un nouveau sous-calque - qu'il n'est possible ni de fusionner avec une autre région ni de subdiviser par une hiérarchie supplémentaire. Or la fusion et la hiérarchisation de calques, comme on peut par exemple la réaliser sous Photoshop ${ }^{\varpi}$, ou tout autre système de regroupement, est indispensable pour la synthèse.

Nous pourrions imaginer le développement de nombreuses autres fonctionnalités: il existe seulement la possibilité de créer des «régions fermées» ou des points, mais pas 
encore de traits ou de zones floues sans délimitation. L'outil est pour le moment moins ergonomique que ces "vieux» logiciels que sont Photoshop ${ }^{\circledast}$ ou Illustrator ${ }^{\circledast}$. Les annotations étaient donc fastidieuses à effectuer et pourraient encore gagner en précision.

\section{Perspectives} accessibilité par un simple clic, est également un atout. Cette approche permet une utilisation du fichier réellement pluridisciplinaire: on peut imaginer centraliser toutes les informations relatives au site - géolocalisation, analyses effectuées, photos macros, etc. - sur ce support en ligne sécurisée, que tous les participants du projet pourront amender au fur et à mesure de leur expertise. Combien de relevés 3D et de constats ont été refaits dans des optiques ou des contextes différents et qui ne peuvent ensuite pas être exploités - au moins partiellement réutilisés - par un autre corps de métier, soit parce qu'ils ne sont pas bien connus de tous, soit parce qu'ils ont été perdus, ou encore parce qu'ils ont été effectués sur un support devenu obsolète? La redondance ou l'impossibilité d'exploitation d'anciennes données est un véritable problème que ce logiciel permettrait de résoudre.

39 - L'accès aux données sera également facilité, notamment au moment du suivi : il ne sera plus nécessaire de rechercher par exemple une photo macro dans un dossier si elle est accessible directement sur le fichier 3D en un clic.

40 - Le suivi dans le temps pourra se faire au sein d'un projet Aïoli ${ }^{\circledR}$ lui-même, puisqu'il sera possible d'ajouter une image du même objet, ou d'un point particulier du site à une date ultérieure et constater ainsi l'évolution des altérations.

41 - La possibilité d'« afficher les caméras » qu'offre Aïoli, comme tout autre logiciel de 3D, nous sera alors très utile. Elle nous évitera de devoir indiquer à la main l'emplacement de la photo macro, comme nous le faisions sur Photoshop ${ }^{\circledast}$ en figurant un cadre. indiqués, il sera par exemple possible de s'y référer pour effectuer de nouvelles prises de vues comparatives d'un point sensible à différentes périodes. Le suivi n'en sera que facilité et la comparaison par le biais de la macro-photographie n'en sera que plus fiable.

Enfin, Aïoli ${ }^{\circledast}$ possède d'autres fonctionnalités, notamment un module d'extension de décorrélation par étirement (Decorrelation Stretch ${ }^{23}$ en anglais). Ce mode de visualisation, utilisé par la NASA ${ }^{24}$ pour améliorer les images de Mars Rover, permet de faire ressortir des éléments presqu'invisibles à l'œil et ainsi, d'améliorer la visualisation des sites dont la 
surface est complexe. DStretch sera un outil utile pour l'art rupestre, notamment pour faire ressortir des pictogrammes difficilement lisibles, mettre en évidence des superpositions ou encore masquer des éléments parasites. Parce que cet outil fonctionne en augmentant les différences de teintes, on peut en attendre a priori de meilleurs résultats pour les pictogrammes que pour les pétroglyphes. Néanmoins, l'image DStretch devrait être un mode d'affichage tout à fait intéressant pour l'étude des roches gravées, notamment pour décorréler les nombreuses figures qui se superposent (fig. 19) et distinguer les gravures anciennes des griffures - vandalisme - plus récentes, à l'instar de la tracéologie ${ }^{25}$.

Figure 19

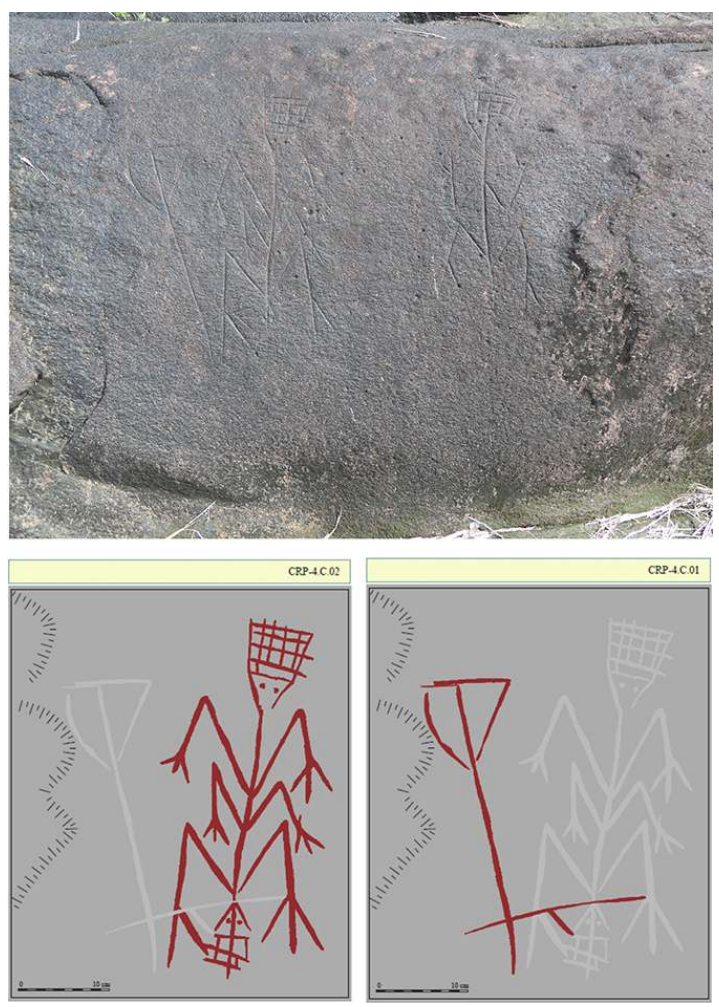

Exemple de deux figures superposées, situées hors carbet, ce qui explique la présence du film biologique épais et sombre en surface gênant d'autant plus la lecture des gravures.

Phot. Anne-Laure Goron. (c) Anne-Laure Goron. Schémas N. Aujoulat. @ CNP.

\section{Conclusion : la 3D, une piste à creuser}

La méthode que nous avons employée, via Photoshop ${ }^{\circledR}$, a l'avantage des calques et des nombreux outils proposés, mais a clairement montré ses limites pour la synthèse, du fait de la grande échelle du site, qui constitue une réelle gageure.

Ce problème d'échelle n'est par ailleurs pas propre à Photoshop ${ }^{\circledR}$. Pour le moment, à notre connaissance, aucun logiciel offrant la possibilité de réaliser des calques et pouvant tourner sur les machines à disposition du grand public ne résout ce problème technique de traiter des objets aussi grands en un seul et même fichier, en raison du poids des fichiers générés. 
Pour couvrir au maximum le développé des roches de la Carapa par le biais de la 2D, environ 100 photos ont été nécessaires. Pour reconstruire la totalité du site en trois dimensions, il en faut au moins 800 . Si la campagne de bêta-test imposait un nombre restreint d'images, Aïoli peut en théorie recevoir un nombre illimité de photos. La durée du traitement de centaines d'images au moment de la création du projet sera le seul facteur limitant.

Le logiciel Aïoli reste donc une solution d'avenir, face aux limites de Photoshop ${ }^{\circledast}$ pour l'élaboration de constats à grande échelle. Nous ne saurions toutefois opposer ces deux méthodes, ou logiciels, qui pourront peut-être un jour se compléter. Et nous espérons que les relevés déjà effectués sur Photoshop ${ }^{\circledast}$ trouveront leur place dans Aïoli .

Quoi qu'il en soit, la possibilité de réaliser des relevés en trois dimensions et de centraliser les données à la fois archéologiques et conservatoires d'un même site offre des avantages considérables en matière de suivi scientifique. Et Aïoli ${ }^{\circledast}$ présente également un intérêt collaboratif. La mise en commun des informations permet de générer des dialogues entre les différentes disciplines et corps de métiers de la conservationrestauration. Le Building Information Modeling ${ }^{26}$ est un processus de plus en plus populaire dans le monde de l'architecture. Aïoli pourrait-il devenir notre BIM adapté au domaine de la conservation-restauration?

\section{BIBLIOGRAPHIE}

AUJOULAT, Norbert. « Le site d'art rupestre de la Carapa ». L'Archéologie en Guyane. Ministère de la Culture, conseil régional de Guyane et APPAAG, 1997, p. 131-141.

AUJOULAT, Norbert. Rapport. La Carapa - Kourou (Guyane) - Corpus des gravures rupestres. CNP/SRA Guyane, 2 vol., 1997.

GASSIES, Éric. « Éléments sur l'art rupestre de la Guyane française. L'art rupestre dans les Caraibes, vers une inscription transnationale en série sur la Liste du patrimoine mondial de l'Unesco ». World Heritage papers, 24, 2008, p. 241-246.

Sources non publiées, archivées à la DAC de Guyane :

ANDRE, Martie-Françoise, BOIVIN, Pierre, GORON, Anne-Laure, ROUSSEL, Erwan. Mission « Roches gravées de Guyane » (20-30 juin 2013), rapport d'étape. DAC de Guyane, 2013.

BOIVIN, Pierre, HENOT, Jean-Marc. Mission « Roches gravées de Guyane » (22-30 juin 2013), étude pétrographique. DAC de Guyane, 18 p., 2013.

BRUNET, Jacques. Note sur la conservation du site amérindien de la Carapa. Kourou. Guyane. SRA Guyane, 4 p., 2004.

CAUTRU, Jean-Pierre. Rapport sur la géologie des roches gravées de la Carapa. SRA Guyane, 4 p., 1993.

GORON, Anne-Laure. Rapport d'intervention - Roches gravées de la Carapa, Kourou, Mission 5 septembre-décembre 2016. DAC de Guyane, 2016. 
GORON, Anne-Laure. Rapport d'intervention - Roches gravées de la Carapa, Kourou, Mission 4 novembre 2015. DAC de Guyane, 2015.

GORON, Anne-Laure. Rapport d'intervention - Roches gravées de la Carapa, Kourou, Mission 3 novembre 2014. DAC de Guyane, 2014.

GORON, Anne-Laure. Rapport d'intervention - Roches gravées de la Carapa, Kourou (aoûtseptembre 2013). DAC de Guyane, 2013.

MAN-ESTIER, Elena, PAILLET, Patrick. L'Art rupestre amérindien de Guyane. Expertises et constats d'état sanitaire. Périgueux : Centre national de préhistoire, 2013.

MAZIERE, Guy \& Marlène. Kourou, Guyane. Les roches gravées de la Carapa. 35 p., 1992.

Sources internet, liens utiles :

- Formation Inp : http://www.inp.fr/Formation-initiale-et-continue/Formation-continue/

Formation-a-distance

- PAO : https://fr.wikipedia.org/wiki/Publication_assistée_par_ordinateur

- Art-Report : http://www.artreport.fr/fr/

- Horus Condition Report : http://horus-conditionreport.com

- CAAK : http://www.ville-kourou.fr/culture/centre-archeologie-amerindienne-de-kourou-caak/

- DAC de Guyane : http://www.culturecommunication.gouv.fr/Regions/Dac-Guyane

- CNP : http://www.culturecommunication.gouv.fr/Thematiques/Archeologie/Centre-nationalde-prehistoire

- MAP de Marseille : http://www.map.cnrs.fr/\#Actu_UMR

- Carbet : https://fr.wikipedia.org/wiki/Carbet_(architecture)

- Photogrammétrie : https://fr.wikipedia.org/wiki/Photogrammétrie

- Décorrélation strech : http://www.dstretch.com/AlgorithmDescription.html

- NASA : https://mars.nasa.gov/mer/gallery/press/opportunity/20040701a.html

- Tracéologie : https://fr.wikipedia.org/wiki/Tracéologie

- BIM : https://fr.wikipedia.org/wiki/Building_information_modeling

\section{NOTES}

1. - Voir le site: http://www.inp.fr/Formation-initiale-et-continue/Formation-continue/ Formation-a-distance [consulté le 03/04/2019].

2. - Voir sur le site : https://fr.wikipedia.org/wiki/Publication_assistée_par_ordinateur[consulté le 03/04/2019].

3. - Voir le site : http://horus-conditionreport.com [consulté le 03/04/2019].

4. - Voir le site : http://www.artreport.fr/fr/ [consulté le 03/04/2019].

5. - Voir le site : https://www.photo.rmn.fr/archive/97-022163-2C6NU0SFJNHL.html [consulté le 03/04/2019]. Et http://www.culture.gouv.fr/public/mistral/joconde_fr? ACTION=CHERCHER\&FIELD_1=REF\&VALUE_1=000SC013142.

6. - Voir le site : https://www.photo.rmn.fr/archive/97-022163-2C6NU0SFJNHL.html [consulté le 03/04/2019].

7. - Voir dans la base Mérimée : notice PA00125427. 
8. - Voir le site : https://fr.wikipedia.org/wiki/Carbet_(architecture) [consulté le 03/04/2019].

9. - BOIVIN, Pierre, HÉNOT, Jean-Marc. Mission «Roches gravées de Guyane (22-30 juin 2013), étude pétrographique ». DAC de Guyane : 2013.

10. - AUJOULAT, Norbert. « Le site d'art rupestre de la Carapa ». L'Archéologie en Guyane. Ministère de la Culture, conseil régional de Guyane et APPAAG : 1997, p. 131-141.

11. - AUJOULAT, Norbert. Rapport. La Carapa - Kourou (Guyane) - Corpus des gravures rupestres. Périgueux : CNP, 1997.

12. - Voir le site : http://www.culturecommunication.gouv.fr/Regions/Dac-Guyane.

13. - Voir le site: http://www.ville-kourou.fr/culture/centre-archeologie-amerindienne-dekourou-caak/ [consulté le 03/04/2019].

14. - ANDRÉ, Marie-Françoise, BOIVIN, Pierre, GORON, Anne-Laure, ROUSSEL, Erwan. Mission "Roches gravées de Guyane» (20-30 juin 2013), rapport d'étape. DAC de Guyane : 2013. (Rapport non publié, disponible aux archives de la DAC de Guyane).

15. - Voir le site : http://www.culturecommunication.gouv.fr/Thematiques/Archeologie/Centrenational-de-prehistoire.

16. - Voir le site : http://www.aioli.cloud/ [consulté le 03/04/2019].

17. - Voir le site : http://www.map.cnrs.fr [consulté le 03/04/2019].

18. - GORON, Anne-Laure. Rapport d'intervention - Roches gravées de la Carapa, Kourou, Mission 5 septembre-décembre 2016. DAC de Guyane : 2016. (Rapport non publié, disponible aux archives de la DAC de Guyane).

19. - En nous appuyant au maximum sur le glossaire de l'ICOMOS.

20. - Voir le site : https://fr.wikipedia.org/wiki/Photogrammétrie [consulté le 03/04/2019].

21. - Nous tenons donc à remercier vivement ces trois institutions et en particulier Éric Gassies, archéologue au SRA de Guyane, chargé entre autres de la conservation des roches gravées de Guyane.

22. - La couverture de prises de vues se fait selon certaines règles pour pouvoir obtenir un bon modèle 3D, notamment le recouvrement des photos de $60 \%$ minimum (1 point doit être vu par 3 photos pour exister) et un cheminement adapté : tourner autour du modèle -24 photos pour $360^{\circ}$; fermer son cheminement - tour complet +3 photos; prévoir des repères de géoréférencement ou d'échelles visibles sur les photos ; faire des zooms sur les zones plus complexes - débords, creux, reliefs.

23. - Voir le site : http://www.dstretch.com/AlgorithmDescription.html [consulté le 04/04/2019].

24. - Voir le site: https://mars.nasa.gov/mer/gallery/press/opportunity/20040701a.html [consulté le 04/04/2019].

25. - Voir le site : https://fr.wikipedia.org/wiki/Tracéologie [consulté le 04/04/2019].

26. - Voir le site: https://fr.wikipedia.org/wiki/Building_information_modeling [consulté le 04/04/2019].

\section{RÉSUMÉS}

À l'heure de la " dématérialisation », le domaine de la conservation-restauration est concerné par l'émergence d'outils numériques mettant notamment l'utilisation de la 3D à la portée de tous. Outre son utilisation pour la restitution d'éléments, qui s'avère prometteuse, la modélisation 3D nous a également intéressée dans le cas des roches gravées de la Carapa (Kourou, Guyane 
française). Ce site, classé monument historique en 1993, a fait l'objet d'une campagne d'étude et de constat d'état sur support numérique entre 2013 et 2017. Son ampleur, le développé important de la roche et la présence d'une couverture empêchant une vue globale du dessus ont rendu cette tâche difficile, les méthodes désormais "classiques" de relevés sur support photo numérique s'avérant chronophages voire inadaptées sous certains aspects. C'est cela qui a motivé l'expérimentation d'un système de relevé et d'annotation sur support 3D développé par le laboratoire MAP (CNRS/MC). Ainsi, grâce au financement de la DAC (Direction des Affaires culturelles) de Guyane et à la collaboration du CNP (Centre national de préhistoire), nous avons pu tester la plateforme "Aïoli » en évaluant son intérêt pour nos travaux de conservationrestauration sur le site de la Carapa. Cet article est un retour d'expérience, mettant d'abord en relief les limites du relevé 2D élaboré sur Photoshop ${ }^{\circledast}$ Elements, puis de quelle façon et dans quelle mesure Aïoli ${ }^{\circledast}$ pourrait y remédier.

In our age of 'dematerialisation', the field of conservation is also affected by the emergence of digital tools, among which 3D modelling is now becoming easily accessible for everybody. In addition to its promising use for the restitution of elements of sculpture or of buildings, 3D modelling also seemed interesting to us in the case of the engraved stones of Carapa, at Kourou in French Guiana. This site, classified as a historic monument in 1993, has been subjected to a full study and to digital condition reports since 2013. The size of the site, the numerous folds of the rock and the presence of a roof made the creation of an overall view from above very difficult. The now 'classic' method involving the annotation of a digital picture proved time-consuming and even inappropriate. This gave rise to the experimentation of a new 3D surveying and annotation system, developed by the CNRS-MAP laboratory (CNRS/MC). Thanks to funding from French Guiana's DAC (Direction of Cultural Affairs) and the collaboration of the CNP (National Centre of Prehistory), we had the chance to test and evaluate the 'Aïoli' platform during our conservation work on the site. This article is an account of that experimentation, highlighting the limitations of the 2D survey undertaken on Photoshop ${ }^{\oplus}$ Elements, then detailing how and to what extent Aïoli ${ }^{\circledR}$ can solve these problems.

\section{INDEX}

Keywords : conservation-restoration, rock engravings, Carapa, French Guiana, Amerindian, PreColombian, wall, photogrammetry, 3D, record, restitution, heritage restoration, tracking, copy, Photoshop, Aïoli

Mots-clés : conservation-restauration, roche, gravures, Carapa, Guyane française, amérindien, précolombien, paroi, photogrammétrie, 3D, relevé, restitution, restauration du Patrimoine, suivi, calques, Photoshop, Aïoli

\section{AUTEUR}

\section{ANNE-LAURE GORON}

Conservateur-restaurateur de sculptures indépendante anlore.g@gmail.com 